\title{
String Breaking and Z(2) Local Symmetry in the 3D Georgi-Glashow model
}

\author{
L. E. Oxman \\ Instituto de Física, Universidade Federal Fluminense, \\ Campus da Praia Vermelha, Niterói, 24210-340, RJ, Brazil.
}

December 18, 2018

\begin{abstract}
In this work, we consider the London limit of the $(2+1) \mathrm{D}$ GeorgiGlashow model with dynamical quarks. Following Polyakov's monopole plasma approach and using dual methods to treat the relevant matter sector, we derive in a clear and straightforward manner the recently proposed gauging of the discrete $\mathrm{Z}(\mathrm{N})$ symmetry of the associated effective vortex theory. Our procedure applies to bosonic as well as to fermionic matter, enabling to derive a useful representation for the Wilson loop and discuss string breaking, due to the creation of dynamical quark-antiquark pairs. This phenomenon corresponds to a perimeter law, a behavior that has been already observed in the lattice.
\end{abstract}

One of the most interesting problems we know at present in Physics corresponds to look for possible phases and understanding confinement in strongly interacting systems like QCD. Then, the search for alternative methods enabling to describe at least the relevant variables that determine the possible phases in the nonperturbative infrared regime becomes a central problem. One of these alternatives corresponds to duality; fundamental progress in this direction has been developed many years ago by A. M. Polyakov and G. 't Hooft [1, 2]. 
In particular, the dual superconductivity scenario 2] can be realized in the framework of the pure $\mathrm{SU}(\mathrm{N})$ Georgi-Glashow model in $(2+1)$ dimensions. Classically, this model contains vortices with topological charge $\mathrm{Z}(\mathrm{N})$. At the quantum level, the vortex sector can be represented by means of vortex operators associated with instanton (monopole-like) singularities in euclidean spacetime, where the vortices are created or destroyed. There are two relevant Green's functions: two-point and $N$-point correlators. They are incorporated by means of the effective lagrangian for the vortex field [2],

$$
\partial_{\mu} \bar{V} \partial^{\mu} V-\mu^{2} \bar{V} V-\lambda(\bar{V} V)^{2}-\beta\left(V^{N}+\bar{V}^{N}\right),
$$

which displays a global $\mathrm{Z}(\mathrm{N})$ symmetry (for a review, with further advances, see [3] and references therein). In the Higgs phase, when the vortex is an elementary excitation, the mass parameter $\mu^{2}$ is positive and there is no spontaneous symmetry breaking of the $\mathrm{Z}(\mathrm{N})$ group. Then, the vacuum expectation value of the vortex field vanishes. However, when the vortices condense, the VEV of the vortex field is nonzero and $\mathrm{Z}(\mathrm{N})$ is spontaneously broken. This leads to domain walls between different possible vacua which for planar systems are string-like. When considering heavy probes, corresponding to a "quark-antiquark" pair, a string is formed in between thus leading to an energy which is linearly increasing with the pair separation, and an area law for the Wilson loop. This phase is then confining.

This nice mechanism continues to be an important topic of research; it is a defying problem with regard to its possible implementation in QCD [2], [4, while many open questions are still present in simplified models, which are also interesting in a condensed matter context [3, 5, 6].

Confining systems in $(2+1) \mathrm{D}$ that incorporate matter in the fundamental representation are not so understood. However, the Wilson loop still provides information about the vacuum response to the presence of a heavy quark-antiquark probe. In particular, a perimeter law for the Wilson loop would imply a constant potential, coming from the creation of dynamical light quark-antiquark pairs out of the vacuum. This phenomenon is called string breaking and has been observed in lattice QCD [7] (using the Polyakov loop) and lattice $(2+1) \mathrm{D}$ models containing bosonic [8] or fermionic [9] dynamical matter. In particular, in ref. [9], string breaking has been detected by means of the Wilson loop criterium.

In ref. [10], by using the vortex condensation approach to the $(2+1) \mathrm{D}$ Georgi-Glashow model, the authors proposed the gauging of the $\mathrm{Z}(\mathrm{N})$ symmetry in eq. (11), when bosonic matter in the fundamental representation 
is included. They also argued that the perimeter law is associated with a boundary effect coming from this gauging.

Besides the Georgi-Glashow model, confinement can be also understood in the case of pure compact $\operatorname{QED}(3)$ [1]. In fact, both models are closely related as, in the London limit, the relevant low energy dynamics of the former is an abelian subgroup of $\mathrm{SU}(\mathrm{N})$, which is compact.

The aim of this letter is following the monopole plasma approach introduced by Polyakov to provide a simple derivation of the $\mathrm{Z}(2)$ gauging phenomenon when dynamical fermionic quarks are present. Also, upon obtaining a dual representation for the Wilson loop, we will discuss how the perimeter law is attained in the limit where the quarks become massless.

Then, our discussion will be focused on compact $\operatorname{QED}(3)$, eventually considered as the London limit of the SU(2) Georgi-Glashow model, when the Higgs and $W^{ \pm}$become very massive. Let us take the following correlation function,

$$
W(s)=\left\langle\exp i \int d^{3} x s_{\mu} f^{\mu}\right\rangle
$$

where $s_{\mu}$ is an external source to probe the system and $f_{\mu}=\varepsilon_{\mu \nu \rho} \partial^{\nu} \mathcal{A}^{\rho}$ is the dual tensor for an abelian gauge field $\mathcal{A}^{\mu}$. The euclidean action associated with our model is,

$$
\int d^{3} x \frac{1}{2} f_{\mu} f^{\mu}+i g \int d^{3} x J_{\mu} \mathcal{A}^{\mu}+K_{F} \quad, \quad K_{F}=\int d^{3} x \bar{\psi}(\not \partial+m) \psi
$$

In the context of the Georgi-Glashow model, $J^{\mu}$ and $\mathcal{A}^{\mu}$ refer to the $\mathrm{SU}(2)$ current and gauge fields along the $\mathrm{U}(1)$ internal direction which is unbroken before compactification, while $\psi$ transforms in the fundamental representation of $\mathrm{SU}(2)$ and is formed by a pair of four component Dirac fields, describing a parity symmetric system.

In particular, note that the correlator (2) will be useful to study the Wilson loop, $W=\left\langle\exp i g \oint d x_{\mu} \mathcal{A}^{\mu}\right\rangle$, integrated over a boundary $\partial \Sigma$, as this loop can be rewritten, by using Stokes theorem, in terms of a $(\Sigma)$ surface integral of the dual tensor. That is, $W=W\left(g \delta_{\Sigma} n\right)$, where $\delta_{\Sigma}$ is a Dirac $\delta$-distribution with support on $\Sigma$ and $n^{\mu}$ is the field of unit vectors normal to $\Sigma$, defined by the surface element $d S_{\mu}=n_{\mu} d S$. This is one of the steps we will need in order to compute the Wilson loop when compactifying the $\mathcal{A}$-sector of our model, as this process is accomplished by first performing 
the replacement,

$$
f^{\mu} \rightarrow f^{\mu}+j^{\mu} \quad, \quad j^{\mu}(x)=g_{m} \int_{\gamma} d y^{\mu} \delta^{(3)}(x-y), \quad g g_{m}=2 \pi,
$$

where $\gamma$ is a Dirac string associated with instanton (monopole-like) singularities located at the string endpoints, and then summing over all the instanton anti-instanton configurations (monopole plasma). In this manner, processes where $\mathcal{A}$-vortices are created or annhilated are incorporated. The vortex magnetic flux is given by the monopole magnetic charge $g_{m}$, which has been taken to saturate Dirac's quantization condition.

Then, we are left with the problem of implementing (4) in the second term of the action (3). For this aim, it is convenient to use a dual transformation of the type introduced in refs. [11, 12, and extensively discussed in refs. 13, 14, 16],

$$
K_{F}+i g \int d^{3} x \mathcal{A}^{\mu} J_{\mu} \leftrightarrow K_{B}+i g \int d^{3} x \mathcal{A}^{\mu} \varepsilon_{\mu \nu \rho} \partial^{\nu} A^{\rho}
$$

(see ref. [14]) where the $\mathrm{U}(1)$ fermion matter current is bosonized using a vector field $A_{\mu}$ whose gauge invariant action is given by,

$$
e^{-K_{B}[A]}=\int \mathcal{D} b_{\mu} e^{-\Gamma[b]-i \int d^{3} x b_{\mu} \varepsilon^{\mu \nu \rho} \partial_{\nu} A_{\rho}} .
$$

The functional $\Gamma[b]=-\ln \operatorname{det}(\not \partial+m+\not b)$ is the effective fermionic action in the presence of an abelian gauge field $b_{\mu}$, along the abovementioned unbroken $\mathrm{U}(1)$ direction. In this regard, note that if we exponentiate the second member in (5) and then use eq. (6), we can path-integrate over $A$ to obtain a $\delta$-functional leading to the constraint, $b_{\mu}=g \mathcal{A}_{\mu}+$ pure gauge. Then, integrating over $b_{\mu}$ we are left with $\exp (-\Gamma[g \mathcal{A}])$, that is, the fermion pathintegral using the first member in (5). Then, the mapping defined by eqs. (5) and (6) is exact. The current bosonization rule $J_{\mu} \leftrightarrow \varepsilon_{\mu \nu \rho} \partial^{\nu} A^{\rho}$ is also universal, and it has been used to discuss the universal transport properties in quantum Hall systems (see ref. [14]). This mapping generalizes the well known bosonization of $(1+1) \mathrm{D}$ fermionic systems, as in that case (5) and (6) are valid with the replacement $\varepsilon_{\mu \nu \rho} \partial^{\nu} A^{\rho} \rightarrow \varepsilon_{\mu \nu} \partial^{\nu} \phi$, that is, the usual bosonization rule for the fermion current, see also ref. [15].

Now, in the $(2+1) \mathrm{D}$ case, because of the current bosonization rule, we can use an integration by parts in the second member of (5) to rewrite the interaction in terms of $f^{\mu}$, thus enabling a proper implementation of (4)). 
Then, in compact $\mathrm{QED}(3)$ with dynamical fermions, the correlator (2) can be represented as,

$$
\begin{gathered}
W(s)=\frac{1}{\mathcal{N}} \int \mathcal{D} j \mathcal{D} A \mathcal{D} \mathcal{A} e^{-S+i \int d^{3} x s_{\mu}\left(f^{\mu}+j^{\mu}\right)}, \\
S=\int d^{3} x \frac{1}{2}(f+j)^{2}+i g \int d^{3} x(f+j) A+K_{B}[A] .
\end{gathered}
$$

The measure $\mathcal{D} j$ represents the path-integral over the monopole plasma, whenever the integrand only depends on the monopole density. See ref. [5], for another possibility to implement the compactification in $\mathrm{QED}(3)$ models with dynamical matter; implications associated with both possibilities are interesting in the context of applications to condensed matter systems [ㅁ, $\underline{6}$.

In our case, we can follow the analysis for pure compact $\mathrm{QED}(3)$ given in ref. [17, adapted for the computation of the correlation function $W(s)$. Introducing a Lagrange multiplier $\lambda_{\mu}$ to linearize the Maxwell term in eq. (8) and integrating over the field $\mathcal{A}^{\mu}$, we obtain the constraint, $g A_{\mu}-\lambda_{\mu}-s_{\mu}=$ $\partial_{\mu} \phi$. Using this information in eq. (8), we get,

$$
W(s)=\frac{1}{\mathcal{N}} \int \mathcal{D} j \mathcal{D} A \mathcal{D} \phi e^{-\int d^{3} x\left(\frac{1}{2}(\partial \phi+s-g A)^{2}+i j^{\mu} \partial_{\mu} \phi\right)-K_{B}[A]}
$$

where we integrate over $\phi$, instead of $\lambda$. Then, the second term can be integrated by parts and written in terms of the monopole density $\rho=\partial_{\mu} j^{\mu}$, so that the integration over $j$ is done over the monopole plasma, thus leading to a sine-Gordon term, $\cos g_{m} \phi$ [1]. After the field redefinition $g_{m} \phi \rightarrow \phi$, and using Dirac's quantization condition $g g_{m}=2 \pi$, we get,

$$
W(s)=\frac{1}{\mathcal{N}} \int \mathcal{D} A \mathcal{D} \phi e^{-\int d^{3} x\left(\frac{1}{2 g_{m}^{2}}\left(\partial \phi+g_{m} s-2 \pi A\right)^{2}-\xi \mu^{3} \cos \phi\right)-K_{B}[A]}
$$

where $\xi$ is the monopole fugacity and $\mu$ is a constant with the dimension of mass. Of course, if matter were not coupled, eq. (10) would read,

$$
W_{0}(s)=\frac{1}{\mathcal{N}} \int \mathcal{D} A \mathcal{D} \phi e^{-\int d^{3} x\left(\frac{1}{2 g_{m}^{2}}\left(\partial \phi+g_{m} s\right)^{2}-\xi \mu^{3} \cos \phi\right)} .
$$

It is worth underlining here that Polyakov's dual field $\phi$ can be identified with twice the phase of the vortex field $V$ (see [3] and references therein). Then, the symmetry $\phi \rightarrow \phi+2 \pi m$ in eq. (11) corresponds to the Z(2) global symmetry of the $N=2$ effective vortex theory in eq. (11). 
When $s_{\mu}=g \delta_{\Sigma} n_{\mu}$, the correlator $W_{0}(s)$ gives the Wilson loop for pure compact QED(3), here represented by means of the celebrated dual action for the field $\phi$ (see ref. 11). According to Polyakov's analysis, the sine-Gordon term introduces a gap, leading to confinement of heavy probes. This comes about from a saddle point evaluation of eq. (11),

$$
\partial_{\mu}\left(\partial^{\mu} \phi+2 \pi \delta_{\Sigma} n^{\mu}\right)-\alpha^{2} \sin \phi=0 \quad, \quad \alpha^{2}=g_{m}^{2} \xi \mu^{3} .
$$

Outside $\Sigma, \phi$ satisfies the sine-Gordon equation and is given by a domain wall containing a $2 \pi$ discontinuity at $\Sigma$, coming from the source term. Therefore, when computing $\left(\partial \phi+g_{m} s\right)^{2}$ in eq. (11), the associated delta singularity in $\partial \phi$ cancels against the $g_{m} s=2 \pi \delta_{\Sigma} n$ term. Away from $\Sigma$, the field is exponentially suppressed. Then, for a large surface, the domain wall leads to an almost uniform action density localized on $\Sigma$ implying the area law for the Wilson loop, that is, confinement.

Now, we see that in the case where the dynamical quarks are coupled, the theory is strongly affected. Namely, the global Z(2) present in eq. (11) becomes local. That is, the symmetry in eq. (10) turns out to be, $\phi \rightarrow$ $\phi+2 \pi m(x), A_{\mu} \rightarrow A_{\mu}+\partial_{\mu} m(x)$, where $m(x)$ is an integer valued function.

According to the discussion in ref. [18, the main operational difference between the local and global versions of a discrete symmetry amounts to a different prescription to carry out functional integrals. In the local case, the fields $\phi$ and $A_{\mu}$ take values in the quotient space. Then, with the equivalence $\phi \equiv \phi+2 \pi, \phi$ must be considered as an angle. As the dual action $K_{B}[A]$ is gauge invariant for any associated transformation $A \rightarrow A-(1 / 2 \pi) \partial \phi$, we can decouple the $\phi$ field in the correlator (10). This also applies to the longitudinal component $\partial \psi$ of the external source $g_{m} s$ appearing in the correlation function (10),

$$
g_{m} s_{\mu}=\partial_{\mu} \psi+R_{\mu} \quad, \quad \psi(x)=-g_{m} \int d^{3} x^{\prime} \frac{\partial_{\mu}^{\prime} s^{\mu}\left(x^{\prime}\right)}{4 \pi\left|x-x^{\prime}\right|},
$$

whenever $\psi$ corresponds to an "angle" field configuration. For the Wilson loop we have, $g_{m} \partial_{\mu} s^{\mu}=2 \pi n^{\mu} \partial_{\mu} \delta_{\Sigma}$ and the values $\psi^{ \pm}$, when we tend to a point on $\Sigma$ from above or below, verify $\psi^{+}=\psi^{-}+2 \pi$, which are identified. Then, we can absorbe the $\partial \psi$ term in $W(s)$ by means of another transformation of the field $A$, and the correlator (10) becomes,

$$
W(s)=\frac{1}{\mathcal{N}} \int \mathcal{D} A e^{-\int d^{3} x \frac{1}{2 g_{m}^{2}}(R-A)^{2}-K_{B}[A]} .
$$


As the system we are considering is parity symmetric (see below eq. (31)), $K_{B}[A]$ does not contain a Chern-Simons term; it only depends on the dual tensor $F=\epsilon \partial A$. Then, transforming $A \rightarrow A+R$ and using eq. (13), the exponent in (14) will contain the term $\int d^{3} x \frac{1}{2 g_{m}^{2}} A^{2}$, plus the bosonized action evaluated on $F+\epsilon \partial R=F+g_{m} \epsilon \partial s$ (cf. eq. (13)) ).

This leads to a qualitatively different situation when compared with the pure gauge result in eq. (11). There, the relevant source $s_{\mu}$ for the Wilson loop is concentrated on a surface, and the localization scale in the dual sineGordon theory implies the area law. On the other hand, when dynamical quarks are present, the local $\mathrm{Z}(2)$ symmetry changes the relevant source to be $\left.\epsilon \partial s\right|^{\mu}=\epsilon^{\mu \nu \rho} n_{\rho} \partial_{\nu} \delta_{\Sigma}$, which is tangent to the boundary of $\Sigma$ and is concentrated there. For instance, in the case of a planar surface on the $x_{3}=0$ plane, the nonzero components are, $+g \delta\left(x_{3}\right) \partial_{2} \Theta_{\Sigma}$ and $-g \delta\left(x_{3}\right) \partial_{1} \Theta_{\Sigma}$, for $\mu=1,2$, respectively, where $\Theta_{\Sigma}=1$, when the transverse coordinates $\left(x_{1}, x_{2}\right)$ are on $\Sigma$, and zero otherwise.

This change in the dimensionality of the source indicates that, depending on the localization properties in eq. (14), a perimeter law could be observed in the phase where dynamical quarks are coupled.

In order to go further, let us analyze the general structure of the fermionic effective action in eq. (6). Again, because of parity symmetry, $\Gamma[b]$ depends on the dual tensor $G=\epsilon \partial b$,

$$
\Gamma[b]=\frac{1}{2} \int d^{3} x G O\left(-\partial^{2}\right) G+\text { nonquadratic part. }
$$

where the nonlocal operator $O\left(-\partial^{2}\right)$ in the exact quadratic part comes from the exact vacuum polarization tensor. For large masses, it can be expanded according to, $O=(1 / m)\left(c_{1}+c_{2}\left(\frac{-\partial^{2}}{m^{2}}\right)+c_{3}\left(\frac{-\partial^{2}}{m^{2}}\right)^{2}+\ldots\right)$. The $c_{i}$ 's are adimensional constants with alternating signs. Because of charge conjugation symmetry, only terms with an even number of external legs contribute to $\Gamma[b]$. So that the nonquadratic part starts with four $G$ factors whose leading term is $1 / m^{5}$. In general, a finite order expansion of $O\left(-\partial^{2}\right)$ presents unphysical poles, which are avoided by the implicit cut-off. This can be already seen at order $1 / \mathrm{m}^{3}$, as $c_{1}>0$ and $c_{2}<0$. However, this can be improved by keeping the full momentum dependence of the polarization tensor (see ref. [13]).

We will consider two important cases where additional information can be given: i) large $m$, where the nonquadratic part in $\Gamma$ is suppressed, ii) $m \rightarrow 0$; in this case, using renormalization group arguments, it has been 
argued that the infrared physics is associated with a nontrivial stable fixed point, dominated by the quadratic term in eq. (15), where $O \sim \alpha\left(-\partial^{2}\right)^{-1 / 2}$ (see ref. [5] and refs. therein). For a related discussion see also ref. [19].

Then, in these limiting situations, the (fixed point) quadratic fermionic effective action, together with eq. (6), leads to a quadratic bosonized action,

$$
K_{B}[A]=\frac{1}{2} \int d^{3} x F\left[-\partial^{2} O\left(-\partial^{2}\right)\right]^{-1} F,
$$

and path integrating in eq. (14), we arrive to an expression that can be used to study the infrared Wilson loop behavior,

$$
W(s)=e^{-\frac{1}{2} \int d^{3} x R\left[g_{m}^{2}+O\right]^{-1} R}=e^{-\frac{g_{m}^{2}}{2} \int d^{3} x \epsilon \partial s\left[\left(-\partial^{2}\right)\left(g_{m}^{2}+O\right)\right]^{-1} \epsilon \partial s} .
$$

In case i), using $O \sim\left(c_{1} / m\right) \rightarrow 0$ in eq. (17), we see that the exponent takes the form $\left(1 / 2 g_{m}^{2}\right) R^{2}$. This result is physically sensible: for any finite probe separation, if we start at a certain mass scale where string breaking occurs, as the fermion mass increases, a situation is expected where the dynamical quarks cannot produce a complete screening anymore. This is manifested through the "magnetic" energy density form of the exponent, and the infinite localization scale in the field $R$,

$$
R=\epsilon \partial\left[-\partial^{2}\right]^{-1} J \quad, \quad J=g_{m} \epsilon \partial s
$$

which prevents the onset of a perimeter law for any given finite loop.

When $m \rightarrow 0$, as discussed, we can still use eq. (16) to analyze infrared behavior. For this aim, let us consider a Wilson loop, running along a large line of size $L$ contained on the $x_{1}$-axis, which then crosses a transverse distance of the order of $L$, goes along a line parallel to $x_{1}$ and crosses back to close the loop. The part on the $x_{1}$-axis gives a nonzero contribution $\left.\epsilon \partial s\right|_{1}=g \delta\left(x_{2}\right) \delta\left(x_{3}\right)$ (see the discussion below eq. (14 $)$ ), whose associated Fourier transform is $g \sin \left(\frac{k_{1} L}{2}\right) /\left(\frac{k_{1}}{2}\right)$. Then, in the exponent of the second member in eq. (17), a typical relevant term giving the leading order behavior in $L$ is,

$$
\frac{\left(g_{m} g\right)^{2}}{2} \int \frac{d^{3} k}{2 \pi^{3}} \frac{1}{k^{2}\left(g_{m}^{2}+\alpha / k\right)}\left[\frac{\sin \left(k_{1} L / 2\right)}{k_{1} / 2}\right]^{2} \approx \frac{L g^{2}}{4 \pi} \int d k_{T}\left(k_{T}+\alpha / g_{m}^{2}\right)^{-1},
$$

where $k_{T}=\left(k_{2}^{2}+k_{3}^{2}\right)^{1 / 2}$. We see that for small $k_{T}$ (large transverse distances) this result is finite, that is, no cut-off of the order of the loop transverse 
dimension is needed. Therefore, when the complete loop is considered, the perimeter law is obtained. This corresponds to string breaking due to the creation of light dynamical quark-antiquark pairs out of the vacuum.

Outside this limiting situations, the quadratic expansion of $\Gamma$ is not in general a controlled procedure to study infrared behavior. Then, for finite masses, it would be interesting to include the effect of nonquadratic terms, in order to gain additional information about the scale for the onset of the Wilson loop perimeter law.

In this work, considering the London limit of the SU(2) Georgi-Glashow model, we have followed Polyakov's monopole plasma approach, together with dual methods for the fermionic matter, to derive in a straightforward manner a useful dual representation for the Wilson loop. In this way, a $Z(2)$ local symmetry is directly evidenced. This gauging is realized in terms of the vector field bosonizing the fermion current, along the U(1) subgroup of $\mathrm{SU}(2)$ which is unbroken before compactification.

Although we have considered fermionic quarks, represented by Dirac fields, our procedure can be also extended to scalar matter, by following the generalized dual methods discussed in ref. [20].

The gauging of the $\mathrm{Z}(\mathrm{N})$ symmetry has been previously proposed in [10], using the vortex condensation approach to the $\mathrm{SU}(\mathrm{N})$ Georgi-Glashow model with scalar matter in the fundamental representation. In that reference, a Wilson loop perimeter law finds an heuristic interpretation in terms of overlaps. Namely, when $\mathrm{Z}(\mathrm{N})$ is local, the Wilson loop operator changes the vacuum inside the loop to a gauge equivalent state, while outside the loop it is left unchanged. Then, the overlap associated with the operator vacuum expectation value is expected to receive nontrivial contributions from a boundary effect.

Here, in the phase where dynamical quarks are coupled, we have seen that the theory displays a local Z(2) symmetry, and the relevant source is indeed concentrated on a boundary. In this phase, when the large mass limit is considered, the scale of localization becomes infinite and the perimeter law cannot be established for any given finite loop, as expected. On the other hand, in the limit $m \rightarrow 0$, an infrared fixed point theory can be used to study the Wilson loop behavior. In this case, a perimeter law is obtained, corresponding to the creation of light dynamical quark-antiquark pairs.

Summarizing, string breaking is a widely observed phenomenon in lattice simulations of $(2+1) \mathrm{D}$ models. Therefore, it is desirable to obtain a 
general theoretical understanding of the subject. Here, we have seen that the consideration of dual methods to treat the dynamical matter constitutes an interesting possibility to deal with compactification and discuss string breaking phases coming from the quark sector.

\section{Acknowledgements}

The author would like to thank C. Schat for useful comments. The Conselho Nacional de Desenvolvimento Científico e Tecnológico (CNPq-Brazil) and the Fundação de Amparo à Pesquisa do Estado do Rio de Janeiro (FAPERJ) are acknowledged for the financial support.

\section{References}

[1] A.M. Polyakov, Phys. Lett. B59, 82 (1975);

A. M. Polyakov, Nucl. Phys. B 120 (1977) 429.

[2] G. 't Hooft, Nucl. Phys. B 138 (1978) 1;

G. 't Hooft, Nucl. Phys. B 190 (1981) 455.

[3] I. I. Kogan and A Kovner, hep-th/0205026.

[4] M. N. Chernodub, F. V. Gubarev, M. I. Polikarpov, V. I. Zakharov, hep-lat/0103033;

N. Seiberg and E. Witten, Nucl. Phys. B 426 (1994) 19;

R. Auzzi, S. Bolognesi, J. Evslin and K. Konishi, Nucl. Phys. B 673 (2003) 187.

[5] I. F. Herbut and B. H. Seradjeh, Phys. Rev. Lett. 91 (2003) 171601;

H. Kleinert, F. S. Nogueira and A. Sudbo, Phys. Rev. Lett. 88 (2002) 232001.

[6] N. E. Mavromatos and S. Sarkar, Int. J. Mod. Phys. B 17 (2003) 251.

[7] C. DeTar, O. Kaczmarek, F. Karsch and E. Laermann, Phys. Rev. D 59 (1999) 031501. 
[8] M. N. Chernodub, E. M. Ilgenfritz and A. Schiller, Phys. Lett. B 547 (2002) 269.

[9] H. D. Trottier, Phys. Rev. D 60 (1999) 034506.

[10] C. D. Fosco and A. Kovner, Phys. Rev. D 63 (2001) 045009.

[11] E. Fradkin and F. A. Schaposnik, Phys. Lett. B 338 (1994) 253;

C.P. Burgess and F. Quevedo, Nucl. Phys. B 421 (1994) 373;

F. Schaposnik, Phys. Lett. B 356 (1995) 39.

[12] E. C. Marino, Phys. Lett. B 263 (1991) 63;

A. Kovner and P. S. Kurzepa, Int. J. Mod. Phys. A 9 (1994) 4669.

[13] D. G. Barci, C. D. Fosco and L. E. Oxman, Phys. Lett. B 375 (1996) 267.

[14] D. G. Barci, L. E. Oxman and S. P. Sorella, Phys. Rev. D 59 (1999) 105012;

D. G. Barci and L. E. Oxman, Nucl. Phys. B 580 (2000) 721.

[15] L. E. Oxman and S. P. Sorella, Phys. Lett. B 531 (2002) 305.

[16] N. Bralic, E. Fradkin, V. Manias and F. A. Schaposnik, Nucl. Phys. B 446 (1995) 144;

J. C. Le Guillou, E. Moreno, C. Nuñez and F. A. Schaposnik, Phys. Lett. B 409 (1997) 257;

R. Banerjee, Phys. Lett. B 358 (1995) 297;

R. Banerjee, Nucl. Phys. B 465 (1996) 157.

[17] D. Antonov, Surveys High Energ. Phys. 14 (2000) 265, hep-th/9909209

[18] L. M. Krauss and F. Wilczek, Phys. Rev. Lett. 62 (1989) 1221.

[19] J. Froehlich, R. Goetschmann and P. A. Marchetti, J. Phys. A 28 (1995) 1169.

[20] C. D. Fosco, V. E. R. Lemes, L. E. Oxman and S. P. Sorella, O. S. Ventura, Annals of Phys. 290 (2001) 27. 